\title{
Considerações sobre Projetos de Filtros Digitais IIR a Partir de Filtros Analógicos
}

\author{
Ricardo S. M. Fernandes, Rui Seara e Sidnei Noceti Filho
}

\begin{abstract}
Resumo-Este artigo discute o uso da transformação espectral para projetos de filtros digitais IIR baseados em técnicas de aproximação para filtros analógicos. É mostrado que a não-linearidade dos parâmetros envolvidos no equacionamento da transformação espectral pode causar distorções significativas na resposta em freqüência do filtro final obtido. Isso ocorre devido à alta sensibilidade numérica dos parâmetros da transformação em algumas situações de aplicação. Estratégias capazes de reduzir tais distorções são apresentadas. Para confirmar a validade do procedimento usado, um exemplo de projeto de filtro é mostrado.
\end{abstract}

Palavras-chave-Filtros digitais IIR, Projeto de filtros, Técnicas de aproximação, Transformação espectral.

Abstract-This paper discusses the use of the spectral transformation for IIR digital filter design based on approximation techniques for analog filters. We show that the nonlinearities involved in the spectral transformation parameters can cause considerable distortions in the frequency response of the obtained final filter. This problem occurs due to the high numerical sensitivity of the transformation parameters in some application situations. Strategies to reduce such distortions are proposed. To confirm the effectiveness of the used procedure, an example of filter design is shown.

Keywords - IIR digital filter, Filter designs, Approximation techniques, Spectral transformation.

\section{INTRODUÇão}

Dentre os possíveis métodos para projeto de filtros digitais IIR, a chamada forma direta se caracteriza por realizar a síntese do filtro desejado diretamente no domínio $z$, quase sempre, fazendo uso de técnicas de otimização numérica. Outras formas de projetos desses filtros são as baseadas na síntese de filtros analógicos, associadas sempre a uma transformação $s \rightarrow z$. Nos dias atuais a síntese de filtros analógicos pode ser realizada através de procedimentos muito bem estabelecidos, utilizando funções de aproximação clássicas. No entanto, da literatura [1], sabe-se que nem sempre as transformações $s \rightarrow z$ funcionam adequadamente.

A obtenção da função de transferência do filtro digital no domínio $\mathrm{z}$ a partir de um filtro analógico pode ser obtida de duas formas:

i) obtenção da função de transferência desejada através de uma transformação direta a partir de sua correspondente função analógica, seja ela passa-baixas (PB), passa-altas (PA), passa-faixa (PF) ou rejeita-faixa (RF). No entanto, dessa forma, o projeto de filtros PA e RF, utilizando alguma técnica de transformação $s \rightarrow z$, não é mais factível para algumas dessas transformações $[1]$;

Ricardo S. M. Fernandes, Rui Seara e Sidnei Noceti Filho, LINSE - Laboratório de Circuitos e Processamento de Sinais, Departamento de Engenharia Elétrica, Universidade Federal de Santa Catarina, Florianópolis, SC, E-mails: \{ricardo, seara, sidnei\}@linse.ufsc.br.

Este trabalho foi parcialmente financiado pelo Conselho Nacional de Desenvolvimento Científico e Tecnológico (CNPq). ii) a outra forma consiste em projetar um filtro PB normalizado, utilizar uma transformação $s \rightarrow Z$ para obter um filtro digital PB protótipo e, através da transformação espectral para filtros digitais [2], [3], obter o requerido filtro PB, PA, PF ou RF desnormalizado. A técnica de transformação espectral proposta em [3] pode ser uma opção atrativa para uso em projetos de filtros digitais IIR considerando (ii). No entanto, devido à alta sensibilidade numérica dos parâmetros da transformação em algumas situações de projeto, distorções significativas na resposta em freqüência do filtro digital final podem ser verificadas. Tal sensibilidade está associada ao mapeamento não-linear dos parâmetros embutido nessa transformação. Assim, neste trabalho, são propostas algumas estratégias de projeto capazes de mitigar tais problemas numéricos nesses casos.

Este artigo está organizado como segue. A Seção II apresenta a descrição do problema. Na Seção III, são tratadas as limitações de cada tipo de transformação (PB/PB, $\mathrm{PB} / \mathrm{PA}, \mathrm{PB} / \mathrm{PF}$ e $\mathrm{PB} / \mathrm{RF}$ ) seguindo uma metodologia de projeto conhecida. Algumas estratégias capazes de reduzir as limitações discutidas são apresentadas na Seção IV. A Seção $\mathrm{V}$ mostra um exemplo de aplicação das estratégias propostas. Finalmente, na Seção VI, são apresentadas as conclusões finais deste trabalho.

\section{DESCRIÇÃo DO PROBLEMA}

A transformação espectral para filtros digitais proposta em [3] consiste de uma transformação algébrica do domínio- $Z$ para um outro domínio- $z$ que modifica a distribuição de freqüência e mantêm inalteradas as características da resposta em magnitude do correspondente filtro digital. Essa transformação é obtida através do mapeamento da variável complexa $Z^{-1}$ na variável complexa $Z^{-1}$, utilizando-se funções unitárias da forma geral seguinte:

$$
Z^{-1}= \pm \prod_{i=1}^{L} \frac{z^{-1}-\alpha_{i}}{1-\alpha_{i} z^{-1}}
$$

onde $\alpha_{i}$ são os parâmetros de mapeamento com $\left|\alpha_{i}\right|<1$. Assim, determinando-se apropriadamente esses parâmetros, pode-se transformar um filtro PB protótipo em um outro tipo de filtro qualquer, seja ele um outro $\mathrm{PB}, \mathrm{PA}, \mathrm{PF}$ ou $\mathrm{RF}$, com características freqüenciais arbitrárias.

No caso de transformações $\mathrm{PB} \rightarrow \mathrm{PB}$ e $\mathrm{PB} \rightarrow \mathrm{PA}$, a função unitária de mapeamento tem ordem 1. Para o caso das transformações $\mathrm{PB} \rightarrow \mathrm{PF}$ e $\mathrm{PB} \rightarrow \mathrm{RF}$, cada singularidade do filtro protótipo é transformada em duas singularidades do filtro desejado. Assim, a função unitária para esses casos é de ordem 2.

Então, para se obter o filtro desejado a partir de um filtro PB protótipo, a definição do tipo de mapeamento e a correspondente determinação dos parâmetros têm um papel fundamental. Como veremos mais adiante, as expressões para determinar os parâmetros de mapeamento são relações trigonométricas um tanto complexas que, para certas condições, apresentam uma sensibilidade numérica elevada, dando origem a erros numéricos importantes; comprometendo, nesses casos, o mapeamento desejado entre os filtros. O resultado é então uma distorção muito acentuada das características do filtro final obtido. 


\section{Metodologia de Projeto}

A primeira etapa do projeto de um filtro digital utilizando transformação espectral é a determinação de um filtro $\mathrm{PB}$ protótipo. De acordo com [2], a escolha da freqüência de corte de banda passante (BP) $\theta_{\mathrm{p}}$ do filtro protótipo PB pode ser arbitrária. Entretanto, conforme a transformação $s \rightarrow z$ utilizada, uma escolha adequada do valor de $\theta_{\mathrm{p}}$ passa a ser muito importante. No caso da transformação bilinear, por exemplo, selecionar um valor de $\theta_{\mathrm{p}}$ mais próximo à freqüência de amostragem $F_{\mathrm{s}}$ pode resultar em uma redução da ordem do filtro protótipo e, conseqüentemente, do filtro desejado.

Uma vez determinado $\theta_{\mathrm{p}}$, pode-se calcular os outros parâmetros do filtro PB protótipo a partir de equações derivadas da função unitária para cada tipo de projeto de filtro. As limitações verificadas na utilização da técnica de transformação espectral ocorrem justamente nessa etapa, na qual estão envolvidas funções não-lineares responsáveis pelo mapeamento $Z^{-1} \rightarrow z^{-1}$.

\section{A. Transformação $P B / P B$}

Esta transformação requer apenas que se alargue ou estreite as bandas de passagem e rejeição do filtro protótipo, mantendo as características de atenuação inalteradas. Conforme [3], a função de transformação PB/PB é dada por

$$
Z^{-1}=\frac{z^{-1}-\alpha}{1-\alpha z^{-1}}
$$

onde $Z^{-1}=\mathrm{e}^{-j \theta}$ e $z^{-1}=\mathrm{e}^{-j \omega}$, com $\theta$ representando as freqüências do filtro protótipo e $\omega$, as freqüências do filtro desejado.

Através da substituição de $Z^{-1}=\mathrm{e}^{-j \theta}$ e $Z^{-1}=\mathrm{e}^{-j \omega}$ em (2) e algumas manipulações algébricas, pode-se determinar o parâmetro $\alpha \mathrm{e}$ a freqüência de corte de banda de rejeição $\theta_{\mathrm{s}}$ do filtro protótipo, a partir das freqüências de corte e de rejeição do filtro desejado $\omega_{\mathrm{p}} \mathrm{e} \omega_{\mathrm{s}}$, respectivamente, e do valor de $\theta_{\mathrm{p}}$ selecionado [2]. Assim,

$$
\alpha=\operatorname{sen}\left[\left(\theta_{\mathrm{p}}-\omega_{\mathrm{p}}\right) \Delta t / 2\right]\left\{\operatorname{sen}\left[\left(\theta_{\mathrm{p}}+\omega_{\mathrm{p}}\right) \Delta t / 2\right]\right\}^{-1}
$$

e

$$
\theta=\frac{1}{\Delta t} \operatorname{tg}^{-1}\left[\frac{\left(1-\alpha^{2}\right) \operatorname{sen}(\omega \Delta t)}{\left(1+\alpha^{2}\right) \cos (\omega \Delta t)-2 \alpha}\right]
$$

onde $\Delta t$ denota o recíproco de $F_{\mathrm{s}}$.

De (3), observa-se que $\alpha$ é uma função não-linear, dependente de $\theta_{\mathrm{p}}, \omega_{\mathrm{p}}$ e $\Delta t$. Na maioria das transformações $s \rightarrow z$ utilizadas, $\Delta t$ deve ser suficientemente pequeno para que não ocorra recobrimento de espectro ou algum outro tipo de distorção no mapeamento entre os domínios analógico e digital.

Sendo assim, fixado $F_{\mathrm{s}}$, a escolha de $\theta_{\mathrm{p}}$ pode não ser tão arbitrária como se havia considerado. Da Fig. 1(a), observa-se que quando $\theta_{\mathrm{p}}$ é menor do que $\omega_{\mathrm{p}}, \alpha$ assume valores entre $[-1,0]$; quando $\theta_{\mathrm{p}}$ é maior do que $\omega_{p}, \alpha$ assume valores entre $[0,1]$; e para $\theta_{p}=\omega_{p}, \alpha=0$.

Então, avaliando o mapeamento $\omega \rightarrow \theta$, através de (4), e assumindo diferentes valores para $\alpha$, observa-se que quanto maior o módulo de $\alpha$, mais não-linear se torna a relação entre as variáveis $\theta$ e $\omega$. Por exemplo, um valor de $\theta_{\mathrm{p}}$ muito alto leva a um valor de $\alpha$ próximo a 1.

Da Fig. 1(b), nota-se que, nessa região, qualquer pequena variação em $\omega$, resulta em uma grande variação em $\theta$.

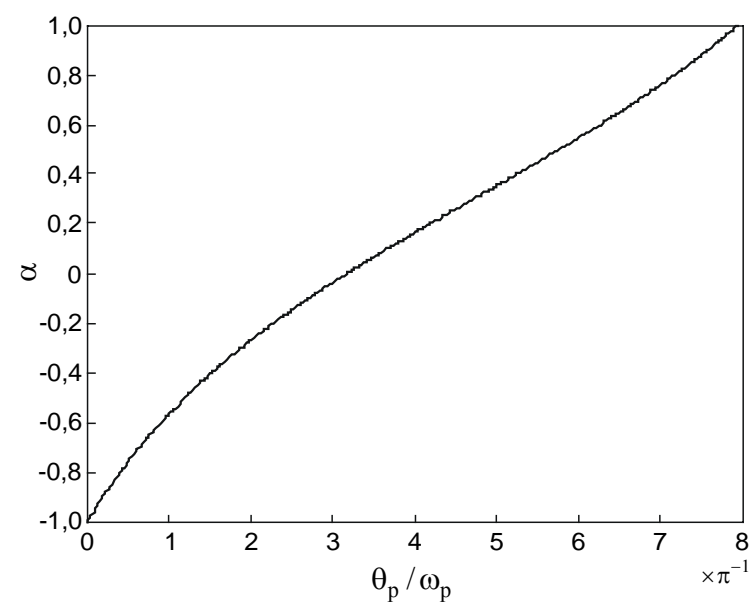

(a)

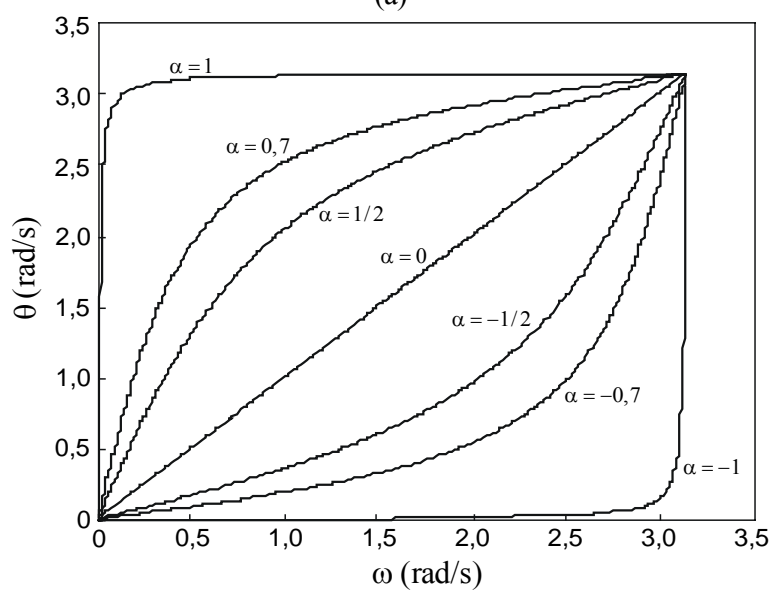

(b)

Fig. 1. (a) Variação de $\alpha \operatorname{com} \theta_{\mathrm{p}}$ relativa a $\omega_{\mathrm{p}}$. (b) Variação de $\theta$ com $\omega$ [conforme (4)] para diferentes valores de $\alpha$ e $\Delta t=1$.

O problema que ocorre aqui é o seguinte: dependendo do valor do parâmetro $\alpha$ utilizado, o mapeamento $\omega \leftrightarrow \theta$ poderá sofrer grandes distorções devido à sua alta sensibilidade numérica, podendo comprometer sobremaneira as características do filtro desejado.

\section{B. Transformação $P B / P A$}

O projeto de um filtro PA digital utilizando transformação espectral pode ser feito de maneira análoga ao projeto de um filtro PB digital. A principal diferença é a função que faz o mapeamento $Z^{-1} \rightarrow Z^{-1}$, ou seja, a transformação do filtro PB protótipo no filtro PA desejado. Tal mapeamento é dado por [3]

$$
Z^{-1}=-\frac{z^{-1}+\alpha}{1+\alpha z^{-1}}
$$

As equações derivadas de (5), utilizadas na determinação de $\alpha$ e $\theta_{\mathrm{s}}$, são, respectivamente,

$$
\alpha=-\cos \left[\left(\theta_{\mathrm{p}}-\omega_{\mathrm{p}}\right) \Delta t / 2\right]\left\{\cos \left[\left(\theta_{\mathrm{p}}+\omega_{\mathrm{p}}\right) \Delta t / 2\right]\right\}^{-1}
$$

e

$$
\theta=\frac{1}{\Delta t} \operatorname{tg}^{-1}\left[\frac{-\left(\alpha^{2}-1\right) \operatorname{sen}(\omega \Delta t)}{\left(1+\alpha^{2}\right) \cos (\omega \Delta t)+2 \alpha}\right] .
$$

Assim como foi feito para a transformação $\mathrm{PB} / \mathrm{PB}$, um estudo do compromisso entre $\theta, \omega$ e $\alpha$, agora para a transformação PB/PA, se faz necessário. Através de (6) e da Fig. 2(a), é possível verificar que $\alpha$ 
novamente depende de $\theta_{\mathrm{p}}$ e $\omega_{\mathrm{p}}$. Se a $F_{\mathrm{s}}$ é feita igual a $2 \pi$, nota-se que, quando $\theta_{\mathrm{p}}$ é menor do que $\pi-\omega_{\mathrm{p}}, \alpha$ assume valores entre $(-\infty$, $-1]$; quando $\theta_{\mathrm{p}}$ é maior do que $\omega_{\mathrm{p}}-\pi, \alpha$ assume valores entre [1, $\infty)$; e quando $\theta_{\mathrm{p}}$ é igual a $\omega_{\mathrm{p}}-\pi, \alpha$ é indefinido.

A Fig. 2(b) mostra o mapeamento $\omega \rightarrow \theta$ para diversos valores de $\alpha$. Nessa figura, observa-se que a região em que o mapeamento é mais linear ocorre para $\alpha$ tendendo a $\pm \infty$. Do mesmo modo, como ocorre para a transformação $\mathrm{PB} / \mathrm{PB}$, quando $\alpha$ assume valores próximos a -1 ou 1 , uma pequena variação de $\omega$ causará uma grande variação em $\theta$.

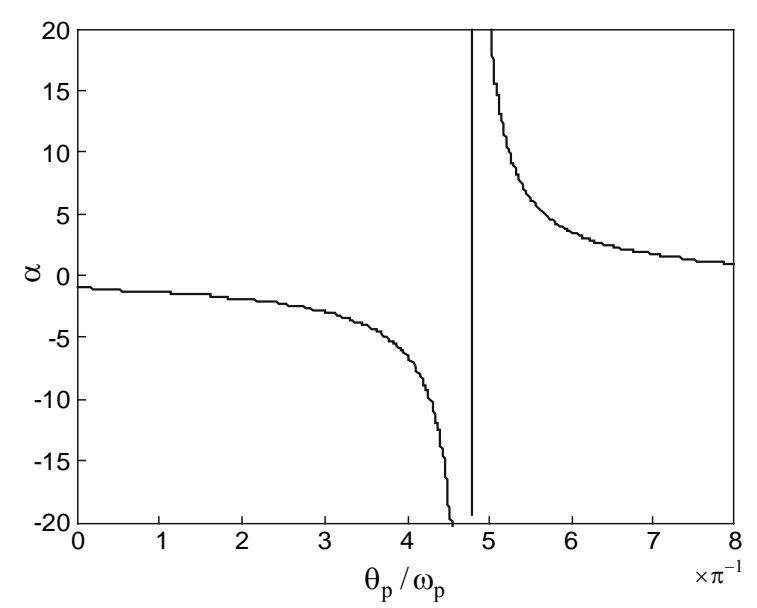

(a)

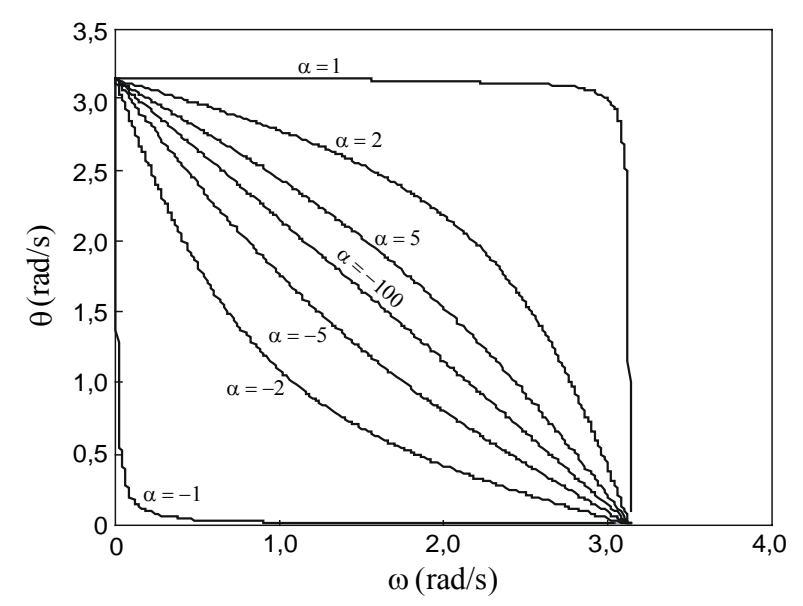

(b)

Fig. 2. (a) Variação de $\alpha \operatorname{com} \theta_{p}$ relativo a $\omega_{p}$. (b) Variação de $\theta$ com $\omega$ [conforme (7)] para diferentes valores de $\alpha$ e $\Delta t=1$.

Assim, para evitar uma possível distorção devido ao mapeamento $\omega \rightarrow \theta$, deve-se escolher uma freqüência $\theta_{p}$ menor do que $\omega_{p}-\pi$, trabalhando-se então com valores negativos de $\alpha$. Conclui-se dessa análise que existe uma região bastante reduzida do espectro de freqüências para a escolha de $\theta_{\mathrm{p}}$ de modo que as limitações de projeto, devido ao efeito de recobrimento de espectro bem como as restrições inerentes à escolha de $\alpha$, atendam às especificações sem que a distorção resultante torne o projeto inviável. Conseqüentemente, existirá apenas uma faixa de valores de $\alpha$ muito limitada para se operar.
Além disso, de acordo com [4], quanto maior a ordem do filtro desejado, maior será a restrição para a escolha de $\theta_{\mathrm{p}}$. Assim, dependendo da transformação $s \rightarrow z$ utilizada e da ordem do filtro desejado, o projeto de um filtro PA, através da transformação espectral, pode se tornar inexeqüível.

\section{Transformação PB/PF}

Neste caso, cada singularidade do filtro protótipo deve ser transformada em duas singularidades do filtro desejado. Como conseqüência, deve-se utilizar agora, conforme mencionado na Seção II, dois parâmetros $(\alpha \mathrm{e} k$ ) para realizar a requerida transformação. Logo, é necessário avaliar a região de linearidade com vistas aos dois parâmetros da transformação. Assim, selecionada a freqüência de corte do filtro PB protótipo $\theta_{\mathrm{p}}$, deve-se determinar os parâmetros $\alpha$ e $k$ da expressão de transformação de segunda ordem, dada por [3]

$$
Z^{-1}=-\frac{z^{-2}-\frac{2 \alpha k}{k+1} z^{-1}+\frac{k-1}{k+1}}{\frac{k-1}{k+1} z^{-2}-\frac{2 \alpha k}{k+1} z^{-1}+1} .
$$

As equações derivadas de (8) usadas para calcular $k, \alpha$ e $\theta$ são, respectivamente,

$$
\begin{gathered}
k=\operatorname{cotg}\left[\left(\omega_{2}-\omega_{1}\right) \Delta t / 2\right] \operatorname{tg}\left[\theta_{\mathrm{p}} \Delta t / 2\right] \\
\alpha=\cos \left[\left(\omega_{2}+\omega_{1}\right) \Delta t / 2\right]\left\{\cos \left[\left(\omega_{2}-\omega_{1}\right) \Delta t / 2\right]\right\}^{-1} \\
\theta=\frac{1}{\Delta t} \operatorname{tg}^{-1}\left[\frac{-4 \alpha k \operatorname{sen}(\omega \Delta t)+2 k \operatorname{sen}(2 \omega \Delta t)}{4 \alpha k^{2} \cos (\omega \Delta t)-\left(k^{2}+1\right) \cos (2 \omega \Delta t)-k^{2}\left(1+\alpha^{2}\right)+1}\right]
\end{gathered}
$$

onde $\omega_{1}$ e $\omega_{2}$ são as freqüências limites da banda passnte e $\omega_{3}$ e $\omega_{4}$ são as freqüências limites da banda de rejeição de um filtro PF. Pode-se obter $\theta_{\mathrm{s} 1}$ e $\theta_{\mathrm{s} 2}$ substituindo em (11) $\omega$, respectivamente, por $\omega_{3}$ e $\omega_{4}$. A freqüência de corte de banda de rejeição do filtro PB protótipo pode então ser obtida por

$$
\theta_{\mathrm{s}}=\min \left(\left|\theta_{\mathrm{s} 1}\right|,\left|\theta_{\mathrm{s} 2}\right|\right)
$$

Nota-se de (9) que $\theta_{\mathrm{p}}$ afeta apenas o valor de $k$, não influenciando o parâmetro $\alpha$. Dessa forma, avalia-se primeiramente o mapeamento $\omega \rightarrow \theta$ em função de $k$. A partir de (9), é possível perceber que $k$ pode assumir valores entre $[0, \infty)$ dependendo do $\theta_{\mathrm{p}}$ considerado, sendo que quanto maior o valor de $k$, maior será o valor de $\theta_{\mathrm{p}}$ e menos linear será a relação entre esses dois parâmetros.

Avaliando o comportamento do mapeamento $\omega \rightarrow \theta$ com respeito a variação de $k$, observa-se da Fig. 3(a) que $k=1$ é o valor mais adequado. Tal situação é descrita em [3], simplificando consideravelmente o equacionamento da transformação espectral. Assim,

$$
Z^{-1}=-\frac{z^{-1}\left(z^{-1}-\alpha\right)}{1-\alpha z^{-1}}
$$

e

$$
\theta=\frac{1}{\Delta t} \operatorname{tg}^{-1}\left[\frac{-2 \alpha \sin (\omega \Delta t)+\sin (2 \omega \Delta t)}{2 \alpha \cos (\omega \Delta t)-\cos (2 \omega \Delta t)-\alpha^{2}}\right] .
$$

A equação de $\alpha$ (10), por sua vez, depende, além da BP do filtro desejado, também do $F_{\mathrm{s}}$ utilizado. Assim, conclui-se que ao se escolher 
$k=1$, a viabilidade do projeto de um filtro PF digital dependerá unicamente do valor de $F_{\mathrm{s}}$ considerado. Como nem sempre é possível se operar com o $F_{\mathrm{s}}$ mais adequado, algumas técnicas de transformação $s \rightarrow z$ podem não ser mais viáveis para esse tipo de projeto. Fazendo-se então a análise do mapeamento dado em (13), para alguns valores de $\alpha$, é possível verificar através da Fig. 3(b) que o valor mais apropriado é $\alpha=0$.

Aparte das limitações descritas acima, outro problema do projeto de filtros PF também foi verificado além da não-linearidade dos parâmetros em relação ao mapeamento $\omega \rightarrow \theta$. A partir da análise das equações e de vários exemplos simulados, constatou-se que quando a banda de passagem do filtro é grande em relação à banda de rejeição, o mapeamento da variável $\omega_{\mathrm{s}}$ gera valores para $\theta_{\mathrm{s}}$ menores que $\theta_{\mathrm{p}}$, tornando impossível o projeto do filtro protótipo. Isso ocorre independentemente da $F_{\mathrm{s}}$ utilizada.

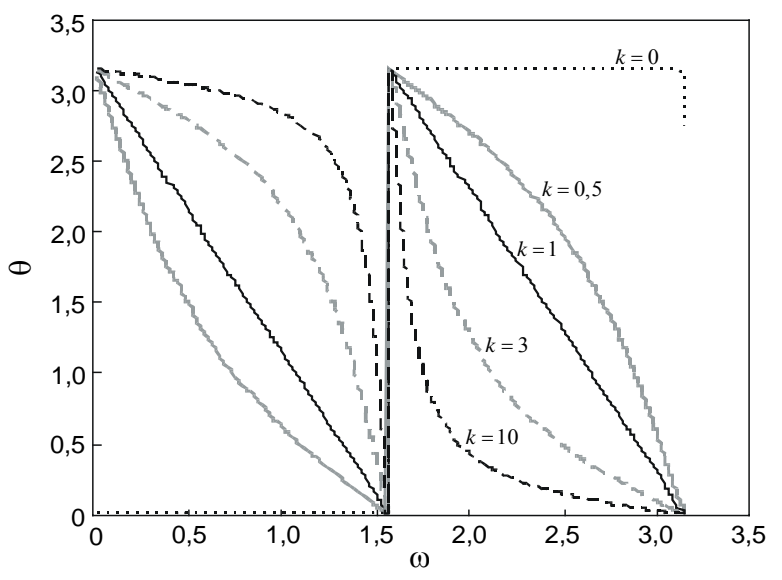

(a)

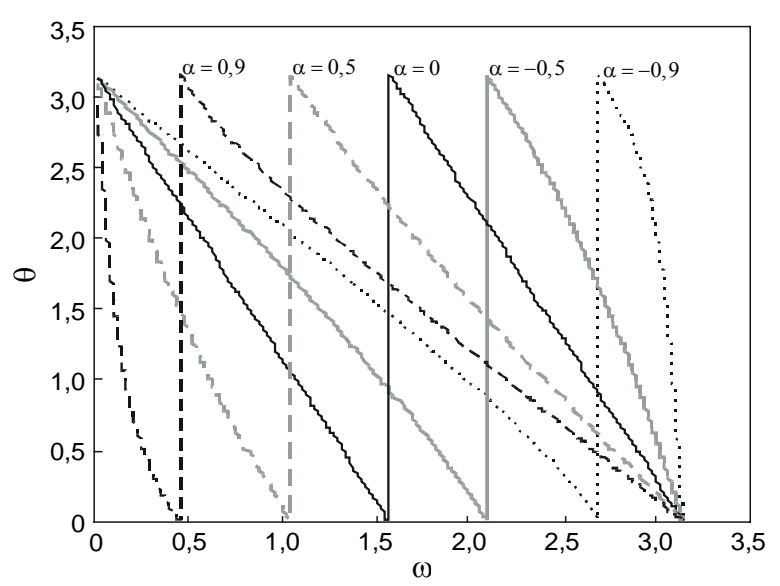

(b)

Fig. 3. Variação de $\theta$ com $\omega$ para $\Delta t=1$. (a) Para $\alpha=0$ e diferentes valores de $k$ [conforme (11)]. (b) Para $k=1$ e diferentes valores de $\alpha$ considerando (14).

\section{Transformação $P B / R F$}

De forma similar ao procedimento usado no projeto de filtros PF, a função de transformação $\mathrm{PB} / \mathrm{RF}$ é também de segunda ordem. Desse modo, faz-se necessário analisar o domínio dos parâmetros $\alpha$ e $k$ em (15).

$$
Z^{-1}=\left[z^{-2}-\frac{2 \alpha k}{1+k} z^{-1}+\frac{1-k}{1+k}\right]\left[\frac{1-k}{1+k} z^{-2}-\frac{2 \alpha}{1+k} z^{-1}+1\right]^{-1} .
$$

Considere que o filtro desejado tenha uma BP limitada pelas freqüências $\omega_{1}$ e $\omega_{2}$ e banda de rejeição limitada por $\omega_{3}$ e $\omega_{4}$. Escolhida a freqüência de corte $\theta_{\mathrm{p}}$ do filtro PB protótipo, pode-se determinar os parâmetros $\alpha$ e $k$ da equação de transformação de segunda ordem [3]. Assim,

$$
\begin{gathered}
k=\operatorname{tg}\left[\left(\omega_{2}-\omega_{1}\right) \Delta t / 2\right] \operatorname{tg}\left(\theta_{\mathrm{p}} \Delta t / 2\right) \\
\alpha=\cos \left[\left(\omega_{2}+\omega_{1}\right) \Delta t / 2\right]\left\{\cos \left[\left(\omega_{2}-\omega_{1}\right) \Delta t / 2\right]\right\}^{-1}
\end{gathered}
$$

e

$$
\theta=\frac{1}{\Delta t} \operatorname{tg}^{-1}\left[\frac{4 \alpha k \operatorname{sen}(\omega \Delta t)-2 k \operatorname{sen}(2 \omega \Delta t)}{-4 \alpha \cos (\omega \Delta t)+\left(k^{2}+1\right) \cos (2 \omega \Delta t)+\left(1-k^{2}\right)+2 \alpha^{2}}\right]
$$

de onde pode-se obter $\theta_{\mathrm{s} 1}$ e $\theta_{\mathrm{s} 2}$ substituindo em (18) $\omega$, respectivamente, por $\omega_{3}$ e $\omega_{4}$. Então, com o auxílio de (12) é possível determinar a freqüência de corte da banda de rejeição do filtro protótipo.

De (16) e (17), constata-se que, assim como para a transformação $\mathrm{PB} / \mathrm{PF}$, neste caso, $\alpha$ também depende apenas da BP do filtro desejado e da $F_{\mathrm{s}}$ utilizada. Já a variável $k$ depende também de $\theta_{\mathrm{p}}$.

Analisando a influência de $k$ no mapeamento $\omega \rightarrow \theta$ (Fig. 4), é possível constatar que $k=1$ é também aqui o valor mais adequado para projeto. Esse valor simplifica sobremaneira o mapeamento $\omega \rightarrow \theta$, como pode ser constatado em (19) e (20); porém, para que isso seja possível, é necessário que $\theta_{\mathrm{p}}=\pi-\left(\omega_{2}-\omega_{1}\right)$.

$$
Z^{-1}=z^{-1}\left(z^{-1}-\alpha\right)\left(1-\alpha z^{-1}\right)^{-1}
$$

e

$$
\theta=\frac{1}{\Delta t} \operatorname{tg}^{-1}\left[\frac{2 \alpha \operatorname{sen}(\omega \Delta t)-\operatorname{sen}(2 \omega \Delta t)}{-2 \alpha \cos (\omega \Delta t)+\cos (2 \omega \Delta t)+\alpha^{2}}\right]
$$

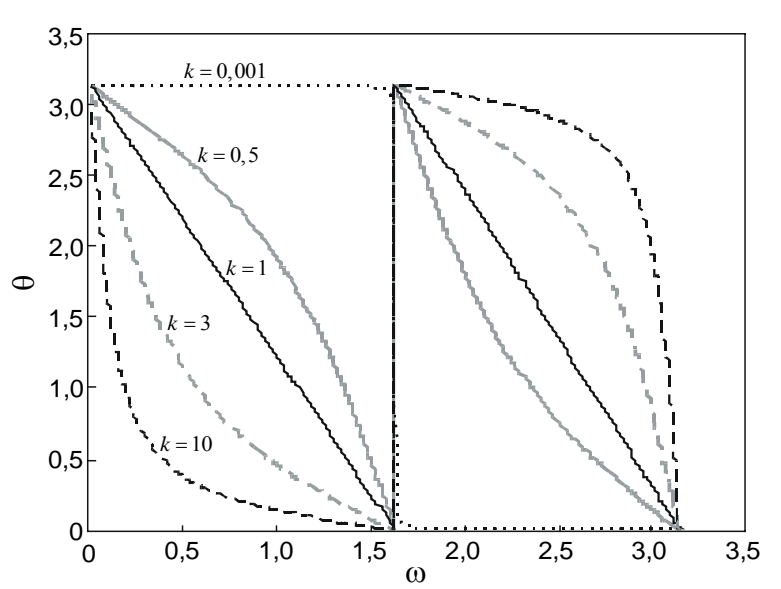

Fig. 4. Variação de $\theta$ com $\omega$ [conforme (18)] para diferentes valores de $k$.

Essa restrição torna o projeto de filtros RF muito mais difícil. Por exemplo, assumindo $k=1$, tem-se uma forte dependência da $F_{\mathrm{s}}$ utilizada. Se $F_{\mathrm{s}}$ for demasiadamente grande, $\theta_{\mathrm{p}}$ estará muito próximo de $\pi$, o que pode causar importante recobrimento de espectro quando, por exemplo, são utilizados os métodos da invariância ao impulso ou da 
invariância ao degrau para o mapeamento analógico/digital. Por outro lado, se $F_{\mathrm{s}}$ for muito pequena, podem-se obter distorções importantes no mapeamento $\omega \rightarrow \theta$ devido ao valor de $\alpha$ usado. A escolha de um $\theta_{\mathrm{p}}$ diferente de $\pi-\left(\omega_{2}-\omega_{1}\right)$, visando reduzir o recobrimento de espectro, pode fazer com que o valor de $k$ escolhido cause alguma distorção no mapeamento $\omega \rightarrow \theta$ e, conseqüentemente, degrade as características do filtro final.

A análise do mapeamento $\omega \rightarrow \theta$ com a variação de $\alpha$, usando $k=1$, é idêntica à realizada para a transformação $\mathrm{PB} / \mathrm{PF}$, visto que (20) é igual a (14), com exceção de um sinal negativo.

Assim como na transformação $\mathrm{PB} / \mathrm{PF}$, o principal problema do projeto de um filtro RF ocorre quando a banda passante do filtro é muito maior do que sua banda de rejeição, pois o mapeamento da variável $\omega_{\mathrm{s}}$ produz valores de $\theta_{\mathrm{s}}$ menores do que $\theta_{\mathrm{p}}$, tornando inexeqüível o projeto do filtro protótipo.

\section{SOLUÇÕEs PROPOSTAS}

Nesta seção, são apresentadas algumas estratégias visando mitigar, em algumas situações, os problemas causados pela sensibilidade numérica elevada dos parâmetros da transformação espectral em projetos de filtros digitais. Um exemplo de projeto de um filtro PA é mostrado. Esse exemplo é obtido a partir de um filtro Cauer analógico seguido de uma transformação $s \rightarrow z$ usando o método da invariância ao impulso. Tal abordagem é escolhida para enfatizar que, mesmo com suas conhecidas limitações para projetos de filtros PA, é possível obter bons resultados considerando as estratégias aqui propostas.

\section{A. Transformação $P B / P B$}

Para este caso, não existem grandes restrições para o projeto deste tipo de filtro digital. A escolha de $\theta_{\mathrm{p}}$ pode ser feita com bastante liberdade e caso venha a ocorrer alguma distorção no mapeamento $\omega \rightarrow \theta$ devido ao valor de $\alpha$ selecionado, isso pode ser corrigido simplesmente escolhendo um valor menor de $\theta_{\mathrm{p}}$.

\section{B. Transformação PB/PA}

Para o caso de um filtro PA, é mostrado que se o mapeamento $\omega \rightarrow \theta$ ocorrer muito próximo a uma região crítica (de forte não-linearidade), isso causará uma severa degradação no filtro desejado. Então, para reduzir tal degradação, é necessário escolher $\theta_{\mathrm{p}}$ mais próximo a $\pi$, o que pode levar a recobrimento de espectro. A maneira proposta para atenuar esse problema conflitante é reduzir a influência de $\alpha$ no processo. Para tal, sugere-se utilizar, para o projeto, uma cadeia de filtros digitais intermediários. O objetivo dessa estratégia é mitigar a influência da escolha dos valores dos parâmetros, considerando assim mais etapas, o que torna as restrições menos severas em cada uma delas.

A implementação desta estratégia pode ser separada em duas etapas. $\mathrm{Na}$ primeira, é feita uma transformação $\mathrm{PB} / \mathrm{PB}$ escolhendo-se um valor $\theta_{\mathrm{p}}$ adequado (suficientemente pequeno para evitar recobrimento de espectro e operar em uma região mais linear de $\alpha$ ). Na segunda, por envolver uma transformação que atua somente no domínio digital, pode-se escolher valores de $\theta_{\mathrm{p}}$ buscando apenas trabalhar na região de maior linearidade de $\alpha$. Quanto mais etapas intermediárias são consideradas, maior (menos restrita) é a faixa de valores que $\alpha$ pode assumir. Por outro lado, quanto maior a ordem do filtro a ser projetado, mais restrita será a faixa de valores de $\alpha$.
Portanto, a primeira etapa consiste em obter um filtro intermediário PB com um valor de $\theta_{\mathrm{p}}$ o mais próximo possível de $\pi$. No entanto, como um alto valor de $\theta_{\mathrm{p}}$ pode causar degradação na transformação $\mathrm{PB} / \mathrm{PB}$, sugere-se utilizar um maior número possível de filtros intermediários, visando distribuir o efeito da influência do alto valor do parâmetro a cada um desses filtros.

\section{Transformação PB/PF}

As limitações que surgem no projeto de um filtro PF podem fazer com que seja mais difícil evitar distorções em alguns casos, pois dois parâmetros ( $\alpha$ e $k$ ) estão envolvidos na transformação considerada. A região de maior linearidade de $k$ ocorre para $k=1$, implicando em $\theta_{\mathrm{p}}=\omega_{2}-\omega_{1}$. Já $\alpha$ depende da $F_{\mathrm{s}}$ e das freqüências da BP do filtro, $\omega_{1}$ e $\omega_{2}$. Assim, quanto mais próxima de $\pi$ estiver a BP do filtro desejado, mais linear será o mapeamento $\omega \rightarrow \theta$. Conclui-se então que, nesse caso, a utilização de filtros intermediários, visando deslocar $\theta_{\mathrm{p}}$, não proporciona qualquer benefício, pois sempre é possível trabalhar com um valor de $\theta_{\mathrm{p}}$, tal que $k$ esteja em uma região de maior linearidade. Além disso, $\alpha$ não é influenciado por $\theta_{\mathrm{p}}$. A maior limitação ocorre quando a $\mathrm{BP}$ não está próxima o suficiente de $\pi$. Isso faz com que $\alpha$ assuma valores que causam degradações quando usada a transformação $\mathrm{PB} / \mathrm{PF}$, pois $\alpha$ depende das freqüências $\omega_{1}$ e $\omega_{2}$. Como essa restrição torna-se mais severa à medida que a ordem do filtro desejado aumenta, alguns projetos de filtros muito seletivos são inexeqüíveis.

As estratégias propostas neste caso são três e, dependendo das características do filtro PF desejado, uma delas pode ser mais eficiente do que as outras. A primeira realiza o projeto sempre escolhendo $\theta_{\mathrm{p}}=\omega_{2}-\omega_{1} \mathrm{e}$, consequentemente, usando $k=1$. Dessa forma, evita-se qualquer distorção causada por esse parâmetro. No entanto, $\alpha$ pode assumir qualquer valor e levar a possíveis distorções no mapeamento $\omega \leftrightarrow \theta$. A segunda utiliza filtros intermediários com o objetivo de deslocar toda a BP do filtro desejado $\omega_{1}$ e $\omega_{2}$, para freqüências próximas a $\pi$, buscando realizar o mapeamento em uma região de maior linearidade. Isso é obtido através de transformações PF/PF para que a banda de interesse do filtro PF possa ser "deslocada" para mais próximo de $\pi$. Tais transformações são realizadas utilizando o mesmo equacionamento da transformação $\mathrm{PB} / \mathrm{PB}$. Assim como na estratégia utilizada para a transformação $\mathrm{PB} / \mathrm{PA}$, é possível utilizar mais de um filtro intermediário de modo a distribuir o efeito da influência do valor do parâmetro entre esses filtros.

A terceira solução separa o projeto do filtro PF em dois: um PB e um PA. A associação em cascata dos dois filtros deverá resultar no PF desejado. Essa solução, entretanto, pode não ser eficaz no caso em que os pólos e os zeros dos dois filtros estejam muito próximos, pois sua influência mútua causa um comportamento indesejado na função final.

\section{Transformação $P B / R F$}

Fazendo a análise das restrições impostas pelos parâmetros da transformação espectral PB/RF discutida na Seção III-D, observa-se que, apesar da semelhança no projeto entre um filtro PF e um RF, algumas características específicas do filtro RF fazem com que nem todas as estratégias aplicadas ao caso anterior se apliquem com sucesso também aqui. É visto que para trabalhar com $k$ em uma região de maior linearidade, é necessário considerar $\theta_{\mathrm{p}}=\pi-\left(\omega_{2}-\omega_{1}\right)$. Assim, quanto mais afastada estiver a BP $\left(\omega_{2}-\omega_{1}\right)$ de $\pi$, maior será o valor de $\theta_{\mathrm{p}}$, 
podendo levar ao recobrimento de espectro em muitos casos. Além disso, outro problema que pode ocorrer, quando o valor de $\left(\omega_{2}-\omega_{1}\right)$ é grande, é o mapeamento de $\theta_{\mathrm{s}}$ em uma freqüência inferior a $\theta_{\mathrm{p}}$. Isso torna inviável o projeto do filtro PB protótipo. Por esses motivos, fica muito difícil se trabalhar com valores de $k$ em uma região de maior linearidade. Tenta-se, então, escolher um $\theta_{\mathrm{p}}$ de modo a operar com um valor de $k$ em uma região onde sua não-linearidade afete minimamente o mapeamento $\omega \rightarrow \theta$. Utilizando filtros intermediários RF/RF, visando abrandar as variações dos parâmetros entre etapas (como descrito para o projeto de filtros PF), é possível reduzir um pouco as degradações no filtro final.

A estratégia de utilizar um filtro PB cascateado com um PA não pode ser aplicada neste caso. Isso porque o filtro $\mathrm{PB}$ necessário possui ao menos um zero no infinito, o que causa uma atenuação indesejada na BP superior do filtro RF. Da mesma maneira, o filtro PA apresenta ao menos um zero na origem, o que causa uma degradação na BP inferior.

\section{EXEMPLO DE APLICAÇÃO}

Considere o projeto de um filtro digital passa-altas a partir de um filtro passa-baixas analógico, usando o método da invariância ao impulso e transformação espectral, com as seguintes características: atenuação na banda passante $A_{p}=1 \mathrm{~dB}$, atenuação na banda de rejeição $A_{s}=50 \mathrm{~dB}$, freqüência de corte de banda passante $f_{\mathrm{p}}=60 \mathrm{~Hz}$, freqüência de corte de banda de rejeição $f_{\mathrm{s}}=50 \mathrm{~Hz}$ e freqüência de amostragem $F_{S}=8000 \mathrm{~Hz}$.

A Fig. 5(a) mostra o resultado de magnitude do filtro digital passa-altas realizado utilizando a transformação espectral aplicada de forma direta. Nota-se que nem as características na banda passante nem as da rejeição atendem às especificações estabelecidas. Nas Figs. 5(b) e 5(c), o projeto é realizado utilizando, respectivamente, um e dois filtros protótipos intermediários. Observa-se claramente que as distorções são consideravelmente abrandadas à medida que mais etapas intermediárias são usadas.

\section{CONCLUSÕES}

Este trabalho discutiu a aplicação da técnica de transformação espectral em projetos de filtros digitais IIR. São levantadas algumas restrições do uso dessa transformação devido à sua alta sensibilidade numérica elevada na obtenção de seus parâmetros em algumas situações de projeto de filtros. Para cada tipo de transformação espectral utilizada, foram levantadas as limitações inerentes, propondo-se algumas estratégias para viabilizar sua utilização. Um estudo da sensibilidade, apresentado em [4], ajuda a clarificar o entendimento dos resultados aqui apresentados. $\mathrm{O}$ exemplo numérico mostrado confirma a eficácia da estratégia proposta para projetos de filtros digitais passa-altas.

\section{REFERÊNCIAS}

[1] A. V. Oppenheim and A. S Willsky, Signals and Systems, 2nd. ed. New Jersey : Prentice-Hall, 1997.

[2] F. L. Perez e R. Seara, "Considerações sobre a utilização da transformação espectral para projetos de filtros digitais a partir de filtros analógicos", Anais do IX Congresso Brasileiro de Automática, Vitória, ES, Set. 1992, pp. 57-62.

[3] A. G. Constantinides, "Spectral transformation for digital filters," Proceedings of the IEE, vol. 117, no. 8, pp. 1585-1590, 1970.

[4] R. S. M. Fernandes, "Projeto de Filtros Digitais Transicionais Cauer-Chebyshev Inverso", Dissertação de Mestrado, Universidade Federal de Santa Catarina, Florianópolis, Brasil, 2006.

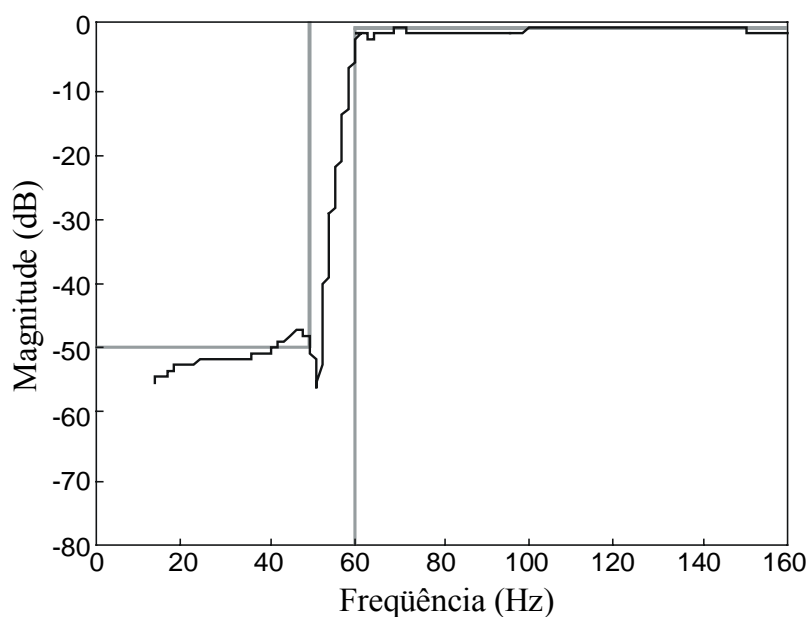

(a)

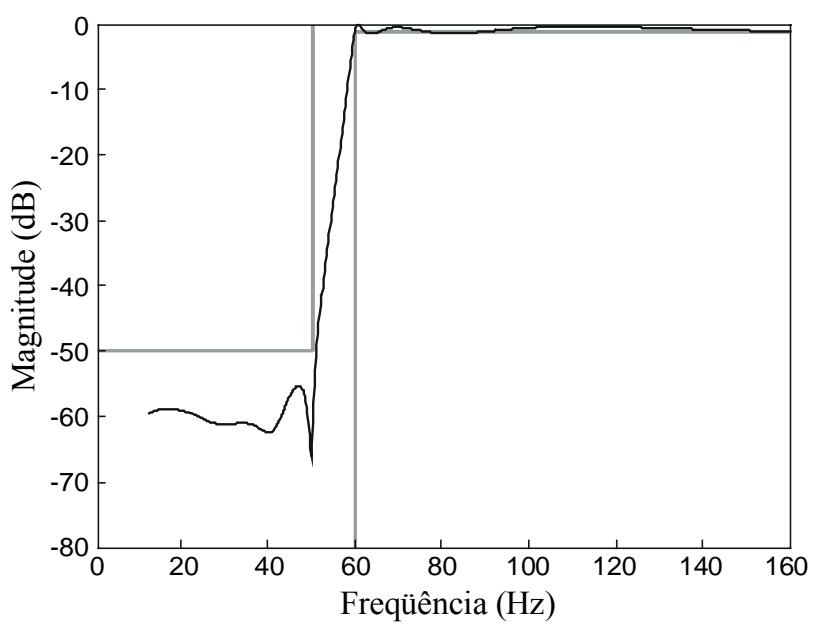

(b)

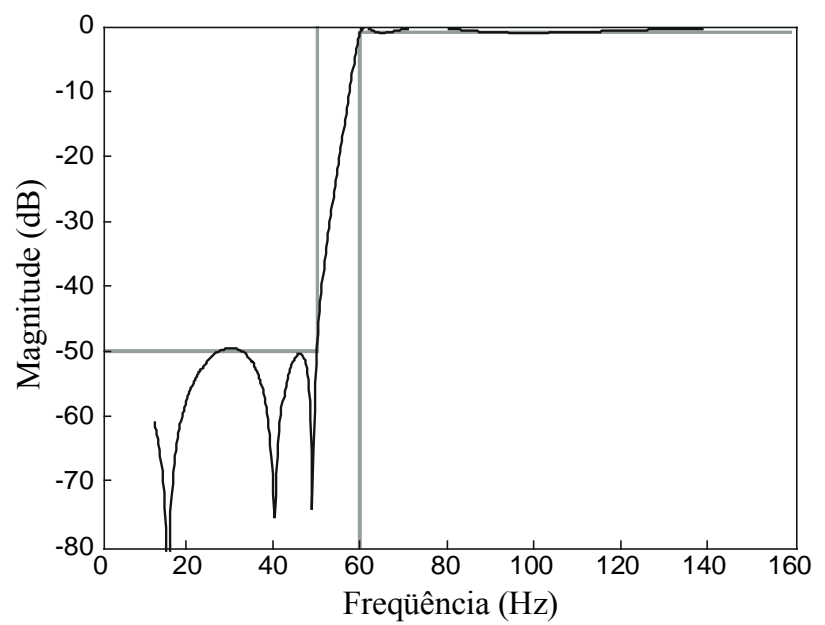

(c)

Fig. 5. Características de magnitude de um filtro digital IIR passa-altas utilizando a técnica de invariância ao impulso associada à transformação espectral. (a) Aplicação direta da transformação espectral (sem uso de filtros intermediários). (b) Usando um filtro intermediário. (c) Considerando dois filtros intermediários. 